Proc. Indian Acad. Sci. (Chem. Sci.), Vol. 105, No. 6, December 1993, pp. 619-628.

(C) Printed in India.

\title{
Studies of photochemical reaction by CIDNP-detected ESR spectrum
}

\author{
Q MENG, Y YAMAKAGE, T AIZAWA, K MAEDA and T AZUMI* \\ Department of Chemistry, Faculty of Science, Tohoku University, Sendai 980, Japan
}

\begin{abstract}
CIDNP-detected ESR spectroscopy was applied to the study of photochemical reaction. In the CIDNP-detected ESR spectroscopy, the spectrum due to intermediate radical pairs and the spectrum due to free (escaped) radicals can be separately observed. The spectrum due to radical pairs is called stimulated nuclear polarization (SNP), and the spectrum due to free radicals is called dynamic nuclear polarization (DNP). The method was applied to the study of photochemical reaction of 2,6-dichlorobenzoquinone and the isomerization of quadricyclane assisted by photoinduced electron transfer, and the chemical species of the intermediate radical pair and of the radical were clarified.
\end{abstract}

Keywords. CIDNP-detected ESR; dynamic nuclear polarization; dichlorobenzoquinone; quadricyclane.

\section{Introduction}

CIDNP-detected ESR spectroscopy is a device to detect ESR spectra of transient free radicals and radical pairs by monitoring the effect of microwaves on the CIDNP spectra of reaction products. The novel aspect of this method is that the ESR spectra of radical pairs and of free radicals can be separately measured (Bagryanskaya et al 1986). The nuclear polarization created by the ESR transitions of radical pairs is called stimulated nuclear polarization (SNP), and nuclear polarization created by the ESR transition of free radicals is called dynamic nuclear polarization (DNP).

The mechanism of SNP is schematically illustrated in figure 1 for a radical pair having two electrons (from radical A and radical B) and one proton. We assume that only radical A has a proton. The figure illustrates the case where the exchange integral $J$ equals zero. The allowed microwave transitions are indicated by vertical lines in the figure. If we could record the ESR spectrum of such a short-lived radical pair, the spectrum would look like the one shown in the right upper corner of the figure. That is, the spectrum consists of two hyperfine lines for radical A and one line for radical $B$. However, it is absolutely impossible, at this stage, to record the ESR of such short-lived species. In the CIDNP-detected ESR spectroscopy, we monitor the CIDNP of either the cage product or the escaped product. In the case of the negative hyperfine coupling constant and the triplet precursor, as is shown in figure 1 , the microwave-induced change of the NMR would be $E / A$ (emission in lower field and absorption in higher field) for a cage product and $A / E$ (absorption in lower field and emission in higher field) for an escaped product. The case of singlet precursor is illustrated in figure 2 . In this case the SNP spectrum should be $A / E$ for a cage product

* For correspondence 
$\mathrm{A}<0 \quad \mathrm{~T}$ - precursor $\mathrm{J}=0$
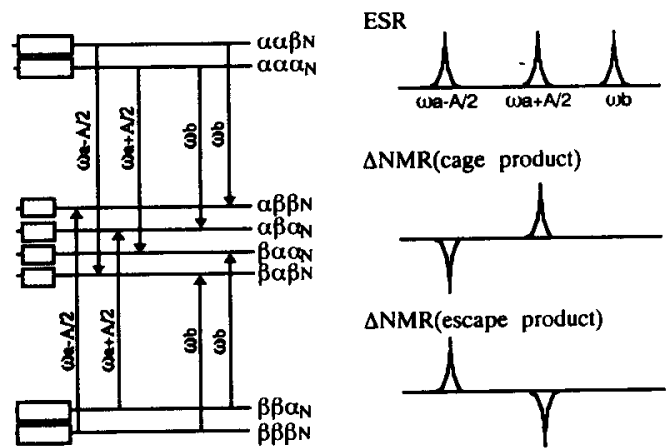

$\triangle$ NMR(cage product)

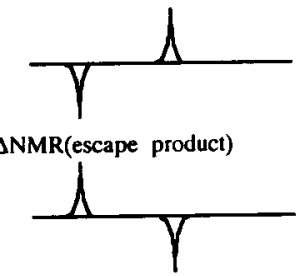

Figure 1. Schematic illustration of the mechanism of stimulated nuclear polarization for the negative hyperfine coupling constant and the triplet precursor.

$\mathrm{A}<0 \quad \mathrm{~S}$ precursor $\mathrm{J}=\mathbf{0}$
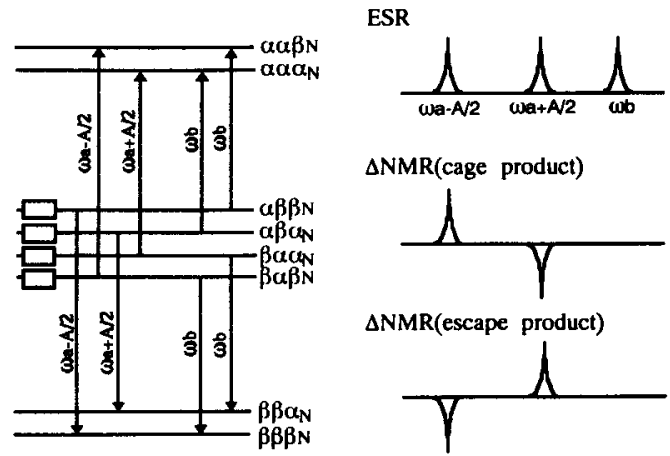

Figure 2. Schematic illustration of the mechanism of stimulated nuclear polarization for the negative hyperfine coupling constant and the singlet precursor.

and $E / A$ for an escaped product. As is understood from these figures, the characteristics of SNP may be summarized as follows.

(1) SNP corresponds to the ESR spectrum of radical pairs.

(2) Sign of polarization differs among hyperfine lines. Thus in the one-proton case shown in figures 1 and 2, the SNP spectrum is either $E / A$ or $A / E$. Thus, the SNP spectrum is symmetric with respect to the center of inversion.

(3) The polarization pattern $(E / A$ or $A / E)$ is determined by (i) precursor spin state,

(ii) reaction product that is monitored, and (iii) microwave power.

The mechanism of DNP is schematically illustrated in figure 3 for a free radical having one electron and one proton. The figure illustrates the cases for both positive and negative hyperfine coupling constants. As is understood from the figure, by the cross relaxation in which total spin quantum number is preserved (i.e. $\Delta m=0$ ) the population transfers from the $\beta_{N}$ nuclear spin state to the $\alpha_{N}$ nuclear spin state in the intermediate radical, thus leading to the enhanced absorption in the NMR of the product (that is, absorptive DNP). By the cross relaxation in which total spin quantum number differs by $2(\Delta m=2)$, on the other hand, the population transfers from the 

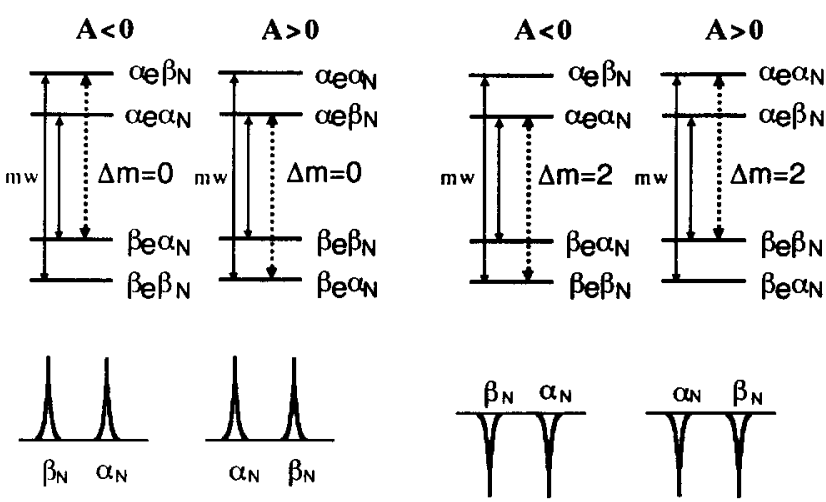

DNP spectra

Figure 3. Schematic illustration of the mechanism of dynamic nuclear polarization.

$\alpha_{N}$ nuclear spin state to the $\beta_{N}$ nuclear spin state leading to the emissive DNP. The characteristics of DNP are summarized as follows.

(1) DNP corresponds to the ESR spectrum of free radicals.

(2) Signs of polarization are the same for all hyperfine lines. For a specific case of one-proton radicals shown in the figure, polarization pattern is either $E / E$ or $A / A$. Thus the DNP spectrum has mirror symmetry.

(3) The polarization pattern (absorption or emission) is determined only by the mechanism of cross relaxation (whether $\Delta m=0$ or $\Delta m=2$ ). It is important to note that the polarization pattern is independent of the sign of the hyperfine coupling constant, contrary to the polarization pattern of CIDNP or of SNP.

As is discussed above, the observed CIDNP-detected ESR spectrum is composed of SNP and DNP. Because of the difference in symmetry (inversion symmetry of SNP and mirror symmetry of DNP) we can easily decompose the observed spectrum into the two components by simulation. (For example, any contribution in the center of the magnetic field should be due to DNP).

We have utilized the technique of SNP and DNP in analyzing various photophysical and photochemical processes. In this paper, however, we discuss only the following two subjects. The first is concerned with the hydrogen abstraction of dichlorobenzoquinone, and the second is related to the isomerization of quadricyclane assisted by photoinduced electron transfer.

\section{Experimental apparatus}

The apparatus for the measurements of CIDNP-detected ESR is schematically shown in figure 4. This apparatus is essentially identical to that reported previously (Meng et al 1990). The sample solution was circulated by a home-made flow system through a sample reservoir, an ESR cavity, and an NMR probe. The sample was irradiated by a $500 \mathrm{~W}$ mercury lamp at the ESR cavity. The products of the chemical reaction were rapidly transferred to the NMR spectrometer, where CIDNP was measured. The transfer rate should be faster than the nuclear relaxation time, which is of the 


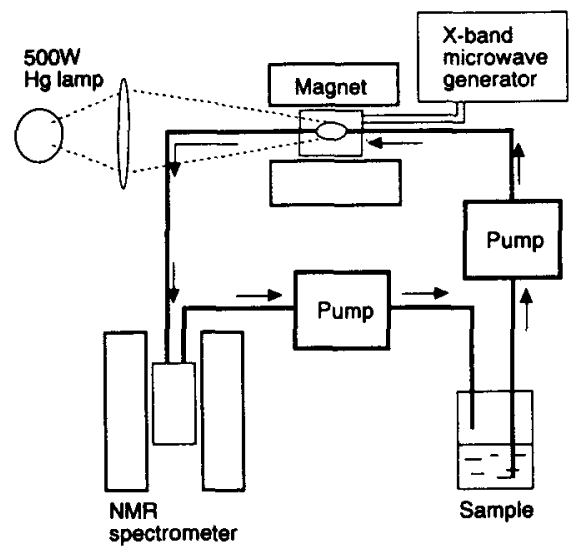

Figure 4. Experimental apparatus for the measurement of CIDNP-detected ESR spectra.

order of a second in the present case. Microwaves amplified to $2 \mathrm{~W}$ by a TWT amplifier were applied at the ESR cavity. The plot of microwave-induced intensity change of CIDNP as a function of the external magnetic field constitutes the CIDNP-detected ESR spectrum.

\section{Hydrogen abstraction reaction of 2,6-dichlorobenzoquinone}

The mechanism of hydrogen abstraction of dichlorobenzoquinone (abbreviated as Q) is schematically shown in figure 5. The mechanism is essentially identical to that of benzoquinone (Pedersen et al 1975; Elliot and Wan 1978). Photoexcited triplet state of $Q$ abstracts hydrogen from a hydrogen donor and the triplet radical pair is produced. The triplet radical pair may dissociate either to semiquinone free radicals or by intersystem crosses to the singlet radical pair. The singlet radical pair may recombine to produce nuclear-polarized $Q$ (denoted by $Q^{*}$ in figure 5). The escaped radicals may undergo further reactions. We note, in the case of dichlorobenzoquinone, that two types of semiquinone radicals, as shown in figure 5 , may be visualized.

This is more specifically illustrated in figure 6. The hydrogen from the hydrogen donor may attack either the oxygen that is close to the chlorine or the one that is far from chlorine. Let us denote the former path as path 1 and the latter path as path 2 . We denote the radical pairs created by paths 1 and 2 , respectively, as radical pairs 1 and 2 . The radicals created by the dissociation of these two types of radical pairs are similarly denoted by radicals 1 and 2 . As is discussed above, the two types of radical pairs may be detected by SNP, and the two types of free radicals by DNP. For this purpose we have measured the CIDNP-detected ESR spectrum.

How we construct the CIDNP-detected ESR spectrum from experimental data is illustrated in figure 7. The spectrum (a) is the ordinary NMR spectrum of the ring proton of $\mathrm{Q}$. Upon irradiation by light, the NMR spectrum becomes like (b); that is, we have absorptive CIDNP. Now, if we further apply microwaves the intensity of the CIDNP is changed. If the external magnetic field is $323.67 \mathrm{mT}$ the CIDNP intensity becomes small as is shown in (c). Thus we have emissive CIDNP-detected ESR signals. If the external magnetic field is shifted to $324.75 \mathrm{mT}$, then the CIDNP 


$$
\begin{aligned}
& \mathrm{Q} \stackrel{\mathrm{hv}}{\longrightarrow} \mathrm{Q} \longrightarrow \stackrel{3}{\longrightarrow} \mathrm{Q} \\
& { }^{3} \mathrm{Q}+\mathrm{RH} \longrightarrow \stackrel{\mathrm{T}}{\longrightarrow \mathrm{QH}} \cdot \overline{\mathrm{R}} \longrightarrow \dot{\mathrm{QH}}+\dot{\mathrm{R}} \\
& \mathrm{S} \frac{\text { isc }}{\mathrm{QH} \cdot \cdot \mathrm{R}} \longrightarrow \mathrm{Q}^{*}+\mathrm{RH} \\
& 2 \dot{\mathrm{QH}} \longrightarrow \mathrm{F} \overline{\mathrm{QH} \cdot \cdot \mathrm{QH}} \longrightarrow \mathrm{Q}^{*}+\mathrm{QH}_{2}(4) \\
& \dot{\mathrm{QH}}+\mathrm{Q} \longrightarrow \mathrm{Q}^{*}+\dot{\mathrm{QH}}
\end{aligned}
$$

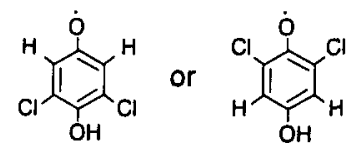

Figure 5. Mechanism of hydrogen abstraction reaction of dichlorobenzoquinone.

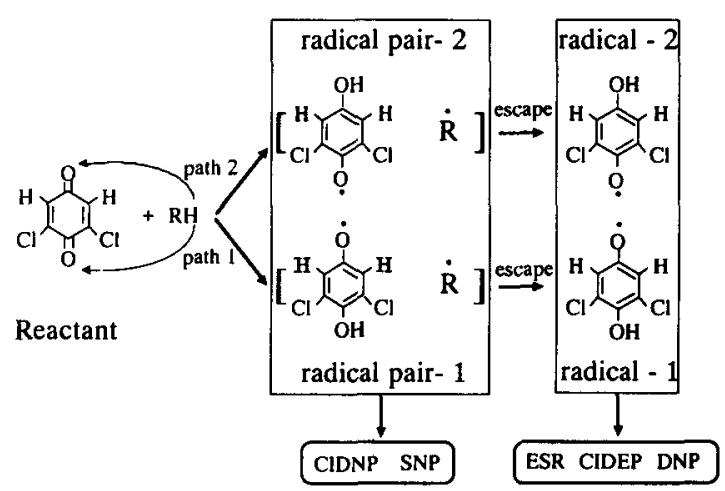

Figure 6. Chemical species that may be present in the hydrogen abstraction reaction of dichlorobenzoquinone.

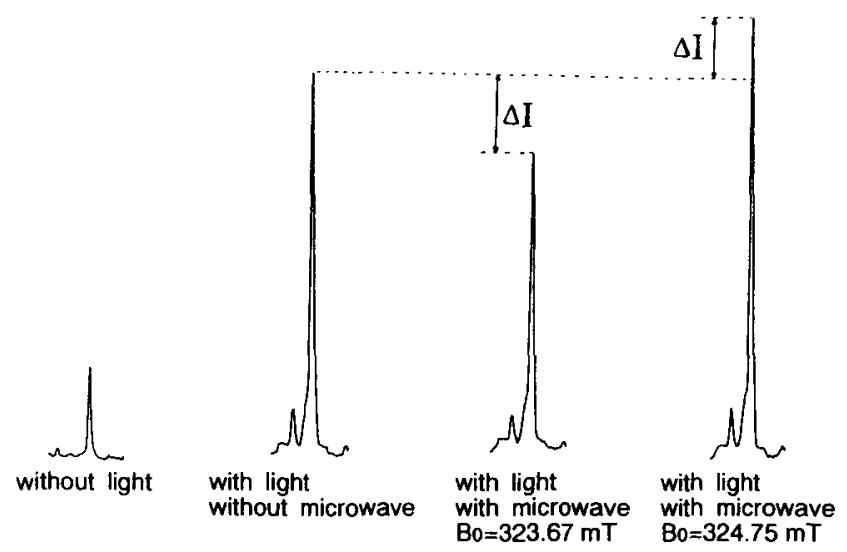

(a)

(b)

(c)

(d)

Figure 7. The NMR spectral data that constitute the CIDNP-detected ESR spectra. 


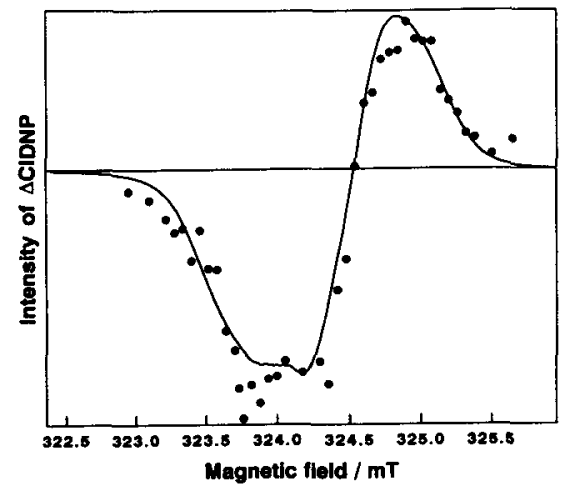

Figure 8. CIDNP-detected ESR spectrum observed during the photolysis of dichlorobenzoquinone. site 1

$(\mathrm{J} \neq 0)$

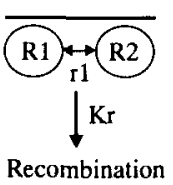

product site 2

$(\mathrm{J}=0)$

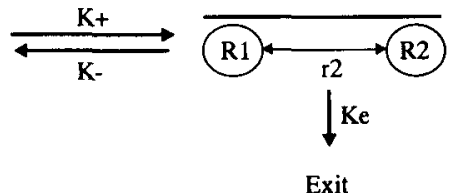

Exit

Figure 9. Illustration of the two-site model in the stochastic Liouville equation.

intensity increases; that is, we have absorptive signals. The CIDNP-detected ESR spectrum is obtained by plotting the microwave-induced NMR intensity change with respect to the external magnetic field.

The observed CIDNP-detected ESR spectrum is shown in figure 8. The dots are the experimental points and the solid curve is the theoretical spectrum calculated in a manner to be discussed below. The spectrum should be composed of four components: SNP of radical pair 1, SNP of radical pair 2, DNP of radical 1, and DNP of radical 2. In order to determine the contributions from the individual components, we try to obtain the theoretical spectra calculated from the stochastic Liouville equation (Koptyug et al 1990).

The stochastic Liouville equation that we are going to solve is as follows:

$$
\mathrm{d} \rho(t) / \mathrm{d} t=-i L \rho(t)+R \rho(t)+W \rho(t)+K \rho(t),
$$

where $L$ is the Liouvillian expressed as

$$
L \rho(t)=H \rho(t)-\rho(t) H .
$$

The spin Hamiltonian is as follows

where

$$
H=H_{0}+H_{1} \text {, }
$$

$$
\begin{gathered}
H_{0}=\mu_{B} \hbar^{-1} B_{0}\left(g_{a} S_{a z}+g_{b} S_{b z}\right)-g_{n} \mu_{N} \hbar^{-1} B_{0} I_{n z}+\sum_{i j}^{a} I_{j} S_{a} \\
+\sum_{j}^{b} I_{j} S_{b}-J(r)\left(\frac{1}{2}+2 S_{a} S_{b}\right)
\end{gathered}
$$

and

$$
H_{1}=\mu_{B} \hbar^{-1} B_{1}\left[\left(g_{a} S_{a x}+g_{b} S_{b x}\right) \cos (\omega t)+\left(g_{a} S_{a y}+g_{b} S_{b y}\right) \sin (\omega t)\right]
$$

The stochastic Liouville equation was solved by a two-site model, which is schematically shown in figure 9. In this model, site 1 represents a radical pair of large $J$ value that may recombine to a recombination product with a rate constant $K_{r}$. 


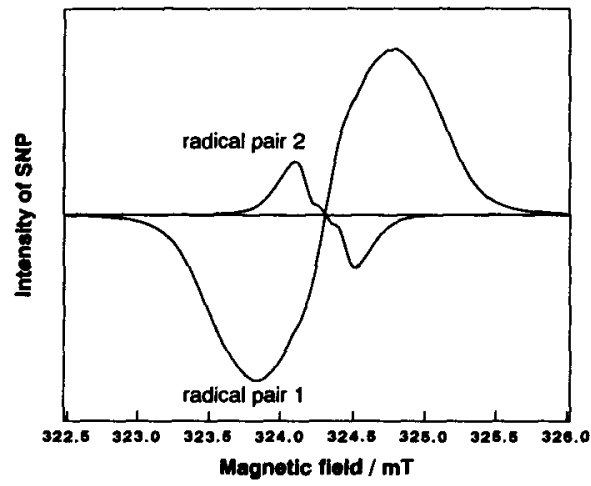

Figure 10. Theoretical SNP spectra for the two types of radical pairs.

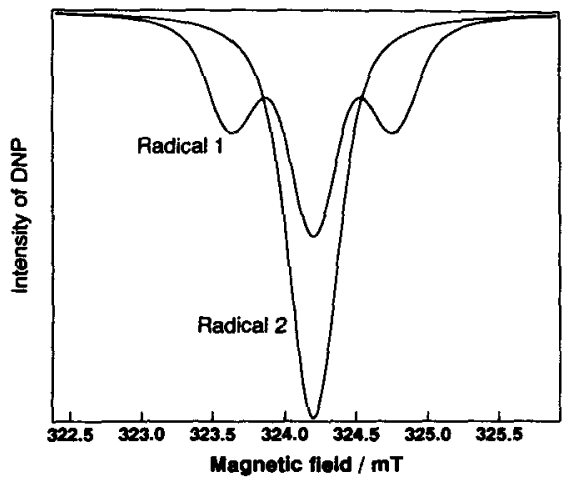

Figure 11. Theoretical DNP spectra for the two types of free radicals.

Site 2 represents the radical pair of $J=0$ and may exit to free radicals with a rate constant $K_{e}$. The transition rate constants between sites 1 and 2 are denoted by $K_{+}$ and $K_{-}$. The parameters that were determined so as to reproduce the structured SNP spectrum of duroquinone are as follows: recombination rate constant $K_{r}=5.0 \times 10^{7} \mathrm{~s}^{-1}$, exit rate constant $K_{e}=3.0 \times 10^{7} \mathrm{~s}^{-1}$, transition rate constants $K_{+}=3.0 \times 10^{6} \mathrm{~s}^{-1}$, and $K_{-}=1.0 \times 10^{5} \mathrm{~s}^{-1}$, exchange integral $J=-5.0 \times 10^{9} \mathrm{rads}^{-1}$. Even though hyperfine coupling constants of radicals 1 and 2 have not been reported, they are determined from the magnetic parameters of $p$-hydroxy-2,3,5,6-tetrachlorophenoxyl radical and p-hydroxyphenoxyl radical (Landolt-Börnstein 1979).

The theoretical SNP spectra thus obtained for radical pairs 1 and 2 are shown in figure 10. We note that the polarization patterns for these two species are different: $E / A$ for radical pair 1 and $A / E$ for radical pair 2 . This is due to the difference in the sign of the proton hyperfine coupling constants. Also, the bandwidth of radical pair 1 is much larger reflecting the larger hyperfine coupling constant. Theoretical DNP spectra for radicals 1 and 2 are shown in frgure 11. These spectra were obtained from simple stick spectra calculated from estimated hyperfine coupling constants for semiquinone radicals multiplied by a Lorentzian line shape.

We try to simulate the observed spectrum in terms of the theoretical spectra of the four species. The results of simulation show that the observed spectrum is well reproduced by the sum of the SNP of the radical pair 1 and the DNP of the radical 2. The contribution from the radical pair 2 and the radical 1 was negligibly small.

The experimental finding discussed above appears to be interpreted only by the mechanism shown schematically in figure 12. That is, the hydrogen from the solvent attacks only the oxygen that is close to the chlorine, producing only radical pair 1. Immediately after radical pair 1 dissociates into radical 1 , the hydrogen (or proton) transfer takes place and only radical 2 is present as free radical. If this mechanism is correct, the ESR and CIDEP spectra should indicate the presence of only radical 2. The steady state ESR and CIDEP spectra (observed at $2.5 \mu$ s after the laser excitation) are shown in figure 13. As is evident from the comparison between the calculated ESR line spectra of the two radicals, the observed spectra are both due to radical 2 which has smaller hyperfine coupling constants. Thus all the experimental evidence appears to support the mechanism outlined above. 


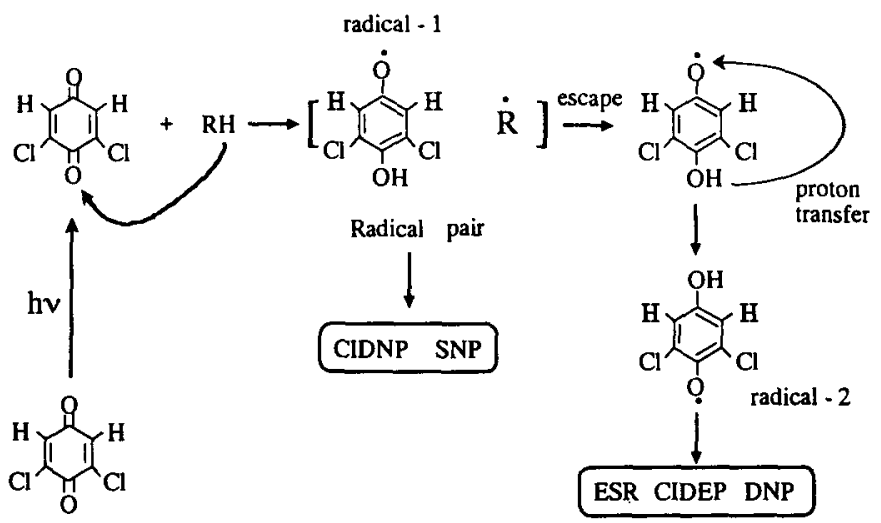

Reactant

Figure 12. Proposed reaction mechanism of the hydrogen abstraction reaction of dichlorobenzoquinone.

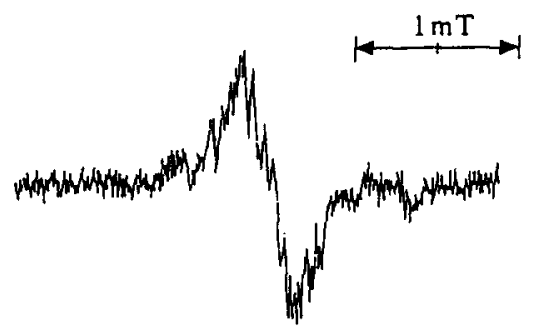

ESR spectrum

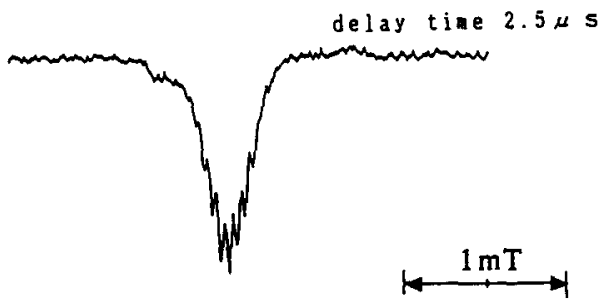

CIDEP spectrum

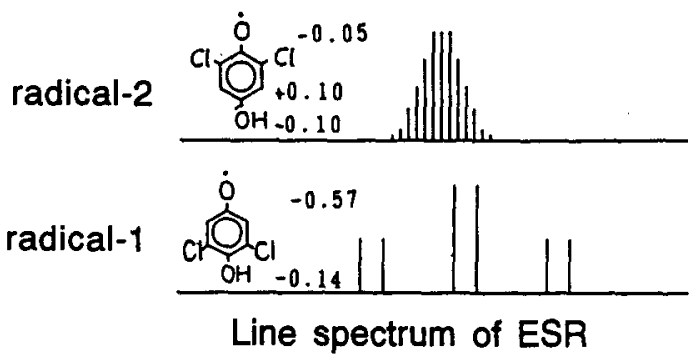

Figure 13. The steady state ESR and the transient CIDEP spectra of radicals produced in the photolysis of dichlorobenzoquinone. 


\section{Photoinduced-electron transfer reaction of quadricyclane}

Quadricyclane is known to isomerize to norbornadiene in the presence of chloranil after photo-induced electron transfer. The reaction is shown in figure 14. A question has arisen as to the species of the intermediate radical pair; is it the cation like I or that like II?

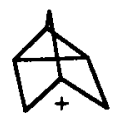

I

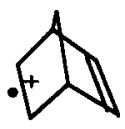

II

This was the question raised by Roth and coworkers sometime ago (Roth and Schilling 1981; Roth et al 1981). They investigated the problem from the viewpoint of the CIDNP spectra and concluded that the intermediate radical pair is composed of the cation I. We try to solve this problem from the viewpoint of the CIDNP-detected ESR spectrum.

The observed CIDNP-detected ESR spectrum in the $L$-band region is shown in figure 15. The signal-to-noise ratio was not necessarily satisfactory; nevertheless, the spectrum clarifies a number of important features. First of all, the spectrum is of the $A / E$ type and the observed CIDNP-detected ESR spectrum is entirely due to SNP.

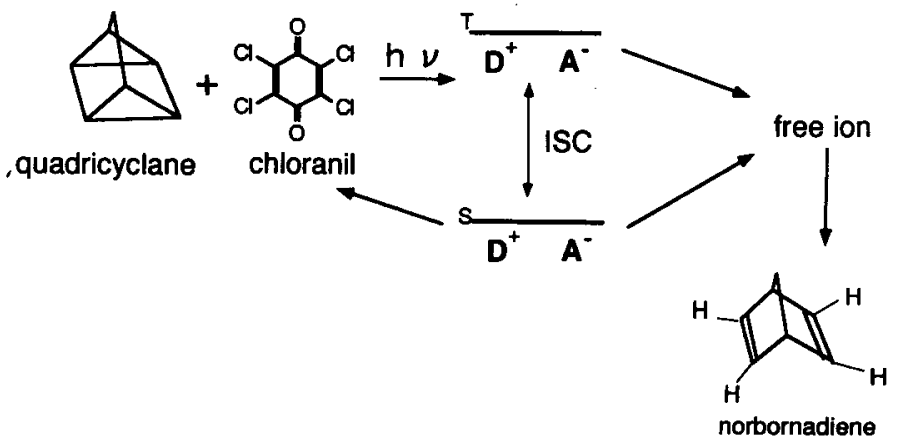

Figure 14. Isomerization of quadricyclane to norbornadiene assisted by photoinduced electron transfer.

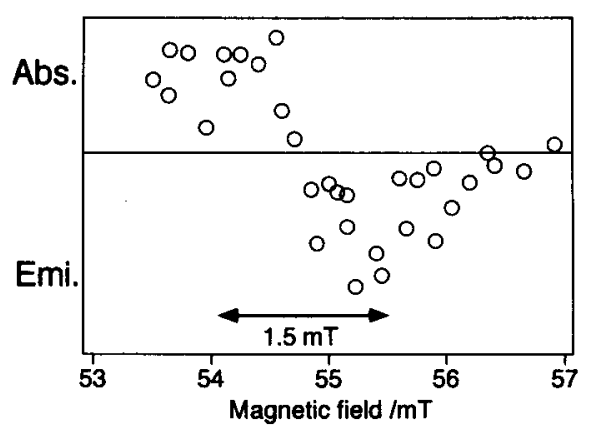

Figure 15. CIDNP-detected ESR spectrum observed during the photolysis of quadricyclane. 
Further, the observed spectrum shows that the whole bandwidth of the SNP is around $1.5 \mathrm{mT}$.

That the $A / E$ pattern was obtained in the case of the triplet precursor, indicates that the hyperfine coupling constant is negative. It is quite important that by SNP the sign of the hyperfine coupling constant can be determined without the knowledge of $\Delta g$ value.

It is interesting to compare our SNP spectrum with the ESR spectrum of the cation radical that was produced by the $\mathrm{X}$-ray irradiation of norbornadiene and quadricyclane. Toriyama and coworkers analyzed the observed ESR spectrum in terms of 4 protons of $0.8 \mathrm{mT}$ and 2 protons of $0.33 \mathrm{mT}$, and they denoted the observed species cation II (Toriyama et al 1983; Nunome et al 1986). The width of $1.5 \mathrm{mT}$ of our observed SNP spectrum is much smaller than the hyperfine coupling constants determined for species II. Thus we conclude that the intermediate radical pair is composed of cation radicals of type $\mathrm{I}$. This conclusion is identical with the conclusion of Roth and coworkers. Even though the conclusion is identical, it is worth pointing out that the species that constitute radical pairs can be determined from the SNP spectrum.

\section{Acknowledgements}

The present research was supported by a Grant-in-Aid for Scientific Research No. 03554013 and a Grant-in-Aid for Scientific Research on Priority Areas "Molecular Based Magnetism" No. 04242102 from the Japanese Ministry of Education, Science and Culture.

\section{References}

Bagryanskaya E G, Grishin Y A, Sagdeev R Z and Molin Y N 1986 Chem. Phys. Lett. 128162 Elliot A J and Wan J K S 1978 J. Phys. Chem. 82444

Koptyug I V, Lukzen N N, Bagryanskaya E G and Doctorov A E 1990 Chem. Phys. Lett. 175467

Landolt-Börnstein 1979 New series; Group II (Berlin: Springer) vol. 9, part C2

Meng Q, Suzuki K, Terazima M and Azumi T 1990 Chem. Phys. Lett. 175364

Nunome K, Toriyama K and Iwasaki M 1986 Tetrahedron 426315

Pedersen J B, Parbo C E M and Muus L T 1975 J. Chem. Phys. 632398

Roth H D and Schilling M L M $1981 \mathrm{~J}$. Am. Chem. Soc. 1037210

Roth H D, Schilling M L M and Jones G II $1981 \mathrm{~J}$. Am. Chem. Soc. 1031246

Toriyama K, Nunome K and Iwasaki M 1983 J. Chem. Soc., Chem. Commun. 1346 